\title{
Tearing mode formation induced by internal crash events at different $\beta_{N}$
}

\author{
V. Igochine ${ }^{1}$, I. Classen ${ }^{2}$, M. Dunne ${ }^{1}$, A. Gude ${ }^{1}$, S. Günter ${ }^{1}$, K. Lackner ${ }^{1}$, \\ R. M. McDermott ${ }^{1}$, M. Sertoli ${ }^{1}$, D. Vezinet ${ }^{1}$, M. Willensdorfer ${ }^{1}, \mathbf{Q}$. Yu ${ }^{1}$, \\ H. Zohm ${ }^{1}$ and ASDEX Upgrade team \\ ${ }^{1}$ Max Planck Institute for Plasma Physics, Boltzmannstr. 2, \\ 85748 Garching, Germany \\ ${ }^{2}$ FOM-Institute DIFFER, Dutch Institute for Fundamental Energy \\ Research, 3430 BE Nieuwegein, The Netherlands
}

E-mail address: valentin.igochine@ipp.mpg.de

\begin{abstract}
Tearing mode formation after internal crash events like sawteeth or fishbones is one of the most important MHD processes that results in a big island structure and associated confinement degradation. The process implies magnetic reconnection at the rational surface, which has been investigated in great detail in the ASDEX Upgrade tokamak. Using direct local measurements it is found that the crash leads to the formation of an ideal kink mode with large saturated amplitude at the resonant surface immediately after the sawtooth crash. This kink mode transforms into a tearing mode on a much longer timescale than the crash itself. The ideal kink mode, formed at the resonant surface after the crash, provides the driving force for the magnetic reconnection. The conversion of the ideal kink mode into a tearing mode after the internal crash is similar for various values of plasma rotation and normalized pressure.
\end{abstract}

Keywords: magnetic reconnection, tearing mode, tokamak

PACS: $52.35 . \mathrm{Vd}$, 52.55.Fa, 52.55.Tn

\section{Introduction}

Magnetic reconnection is a topic of common interest for astrophysical and fusion plasmas [1]. In tokamaks, sawtooth crashes and tearing modes are the most famous events which require magnetic reconnection for their development. During the tearing mode formation, magnetic reconnection rearranges the magnetic topology at the resonant surface with safety 
factor $q=m / n$, where $m$ and $n$ are integer numbers and represent the poloidal and toroidal mode numbers, respectively.

The drive for magnetic islands could dominantly come either from the current profile (classical tearing mode, TM) or from the pressure profile (neoclassical tearing mode, NTM). At the same time, other events in the plasma can also provide the drive for the reconnection during the so-called "forced magnetic reconnection process" and this is one of the main sources for the instability. For example, a large sawtooth crash produces fast relaxations of the core plasma density and temperature and provides this forced drive at the neighbouring resonant surfaces where a tearing mode is created $[2,3]$. The neoclassical tearing mode is metastable and can be triggered by other MHD events via the forced magnetic reconnection at much lower $\beta_{N}$ values compared to the onset value where the seed island grows from noise $[4,5,6,7] .\left(\beta_{N}=\beta\left(a B_{t} / I_{p}\right), \beta=2 \mu_{0}\langle p\rangle / B_{t}^{2} ;\langle p\rangle\right.$ is the volume average pressure, $B_{t}$ is the toroidal magnetic field, $a$ is the minor radius and $I_{p}$ is the plasma current.) Independent of the origin and the drive, the topology of the island structure remains the same in all cases. In case of forced magnetic reconnection, the drive for the tearing mode is provided either by background MHD instabilities (sawteeth, fishbones, ELMs, etc.) or by external magnetic perturbations [8]. When the mode has grown to a critical island width, the pressure profile becomes flat within the island and the neoclassical drive takes over. Small islands are not able to provide significant pressure flattening and might be driven by other mechanisms $[9,10]$. In this paper, only the mechanisms of the seed island formation by drives due to MHD events in the plasma are investigated in detail. This type of tearing mode formation is considered to be the most important for future fusion reactors like ITER [11], because large internal events provide strong magnetic perturbations and are thus able to trigger the mode already at very small normalized pressure values.

In this paper we investigate this seeding process in detail. Previous observations from different tokamaks, for example from JET [12] or TCV [13], report the simple picture of fast tearing mode formation during the crash event and large island widths directly after the crash, based on analysis of magnetic and SXR measurements. Such measurements show large mode amplitudes directly after crashes also in our experiments, but they do not allow the differentiation between simple deformation (ideal kink) and changes of the magnetic topology (tearing mode). It is shown in this paper that a dominantly ideal mode, with the same helicity as the tearing mode, is generated by the crash at the resonant surface and produces this strong signal. The mode converts into a tearing mode only on a longer time scale. This changes the picture of the seeding process considerably and it contradicts the idea of a fast island 
formation during the crash. In some aspects, the situation is similar to advanced tokamak regimes, in which ideal resistive wall modes convert into tearing modes with the same helicity. Such observations were reported by Buratti from JET tokamak [14].

This paper consists of five sections. In section 2, we describe the method of the analysis to distinguish between an ideal kink and a tearing mode with the same helicity. The experimental results for lower beta cases are described in section 3. In section 4, the results for high beta cases are presented. Finally, in section 5 we summarize and discuss the main results.

\section{Experimental identification of the transition from ideal into resistive mode}

In this paper, the following diagnostics are used to investigate the triggering process: (i) magnetic coils, (ii) soft X-ray cameras (SXR) [15], (iii) charge exchange recombination spectroscopy (CXRS) and (iv) two independent electron cyclotron emission (ECE) diagnostics (ECE-Imaging [16] and standard ECE [17]). The ECE diagnostics measure the local electron temperature, $T_{e}$, along radial lines at the same toroidal position. Standard ECE provides local measurements along a single line of sight from the plasma center to the edge. It crosses the $\mathrm{q}=1$ and $\mathrm{q}=2$ resonant surfaces. The ECE-Imaging diagnostic measures the local electron temperature along 14 radial lines of sight with smaller spatial separation $(1.3 \mathrm{~cm})$ compare to the standard ECE. The measurements are located around $q=2$, where the $(m=2$, $\mathrm{n}=1$ ) mode is triggered by sawtooth crashes. Both diagnostics give information about local temperature perturbations inside the plasma for regions of finite background temperature gradient $\left(\delta T_{e}=-\xi \cdot \nabla\left\langle T_{e}\right\rangle\right.$, where $\xi$ is the displacement of the magnetic field lines). The temperature fluctuations are not visible in the case of flat $T_{e}$ profiles and are hardly extractable for cases with strong evolution of the background temperature profile $\left(\left\langle T_{e}\right\rangle\right)$, which is exactly the case directly after a sawtooth crash. In this situation, magnetic coils located outside the plasma provide a good indicator of the total mode amplitude (kink+tearing).

In the experiment, the mode rotates past the ECE detector position. ECE signal variations are used to distinguish between kink and tearing modes. This method and some measurements have already been described in [18], but is briefly summarized here for convenience (see figure 1a). Two measurement points (1) and (2) move along the dashed line during mode rotation. An ideal mode produces sinusoidal temperature variations in all channels around the resonant surface and all of these perturbations are in phase. An island 
has either a flat temperature profile inside or it has an increase towards the centre of the Opoint in our studies (hot island case). This changes the behaviour of the signal (1) that crosses the island separatrix. It contains either a flat region or an additional maximum. The analysis gives a direct indication of the island separatrix position and the character of the mode (kink or tearing). An example is shown in figure 1b for discharge \#27257. After the sawtooth crash, all ECE signals are in phase. At the end of the time window, the island structure is clearly visible in all ECE-Imaging channels from 7 to 2. Backward tracing in time allows us to identify the point in time at which the island feature appears for the first time in each of the channels (indicated by the dashed lines). Magnetic signals show a large amplitude for this ideal $(2,1)$ mode immediately after the sawtooth crash $(\mathrm{t}=2.775$, bottom time trace of figure $1 b)$.

a)
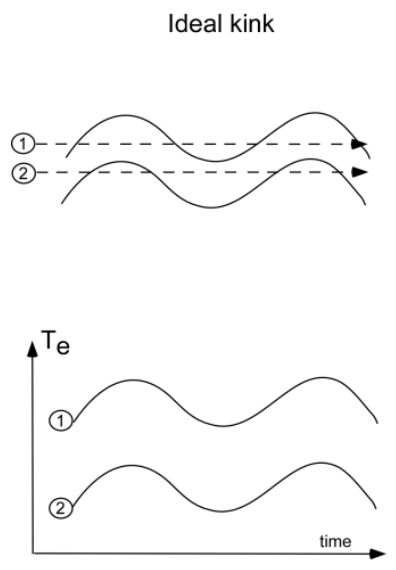
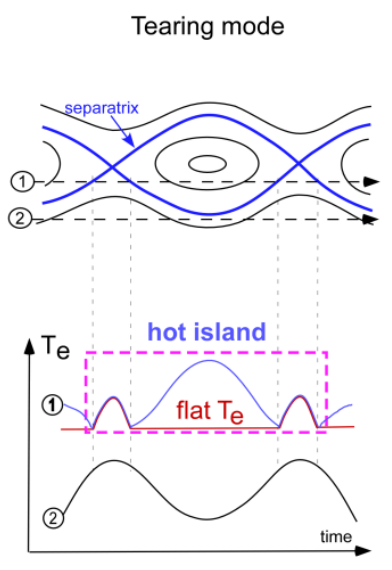

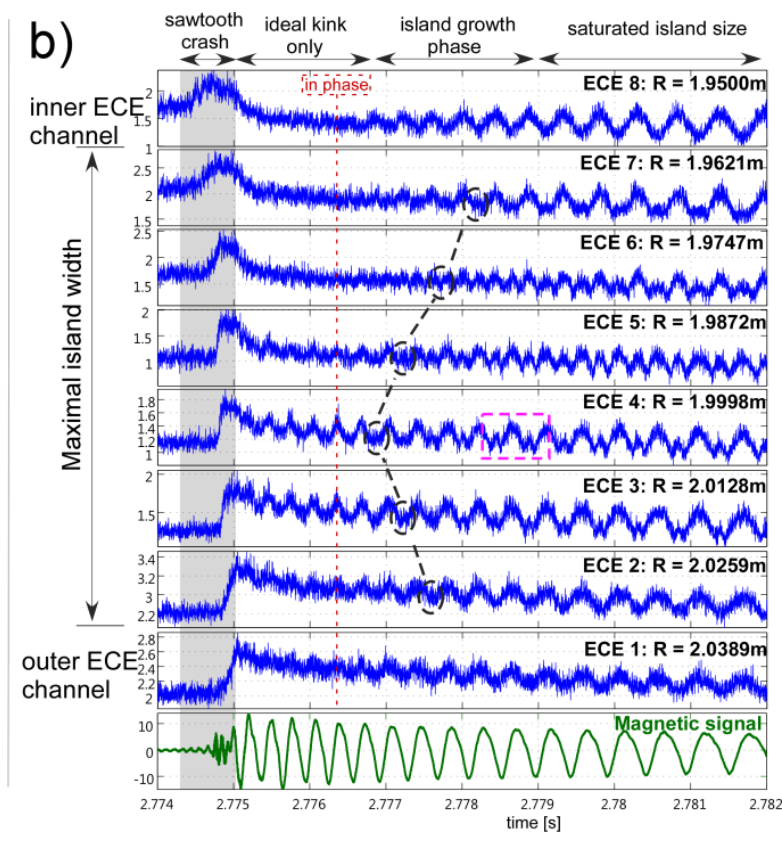

Figure 1. Identification of the mode character from local ECE measurements.

a) Schematic representation of the temperature perturbation for the ideal kink case (left) and island with flat temperature inside and hot island case (right). Separatrix of the island is shown in blue. b) Direct identification of the conversion from ideal kink mode into hot island for discharge \#27257 at $t \approx 2.775 s$. Radial positions of the measurements are given for all ECE channels (vertical position is identical). The bottom time trace is the magnetic signal. The shadow region indicates duration of the sawtooth crash as it is seen by central ECE channel. (Reproduced from [18], with the permission of AIP Publishing.)

In the following, we use this method for island size identification from ECE signals together with other standard methods for the analysis of ECE, SXR and magnetic signals [19]. The advantage of the presented method is its ability to detect an island even if it is so small that it is seen only by a single ECE channel and no pronounced phase jump of the ECE signal can be observed. The total amplitude of the perturbation is extracted from the magnetic signals. Soft 
X-ray tomography is used for the identification of the mode amplitude in the plasma core, for mode number analysis (together with magnetic signals), and in some cases for mode structure identification.

\section{Tearing mode seeding at low $\beta_{N}$}

In this section we discuss tearing mode seeding in L-mode plasmas of ASDEX Upgrade $\left(I_{p}=1 M A ; B_{t}=2.45 T ; n_{e}=4.4 \cdot 10^{19} \mathrm{~m}^{-3} ; q_{95}=4.18\right)$. The case presented in figure $1 \mathrm{~b}$ is the first example of such an event with $\beta_{N}=0.25$ (before the sawtooth crash). Similar analysis is presented for a second case in figure $2 \mathrm{a}\left(\beta_{N}=0.21\right)$. There, the kink-tearing mode transition takes a much longer time. The ideal phase is clearly seen in figure $2 b$, where all perturbations are in phase. 

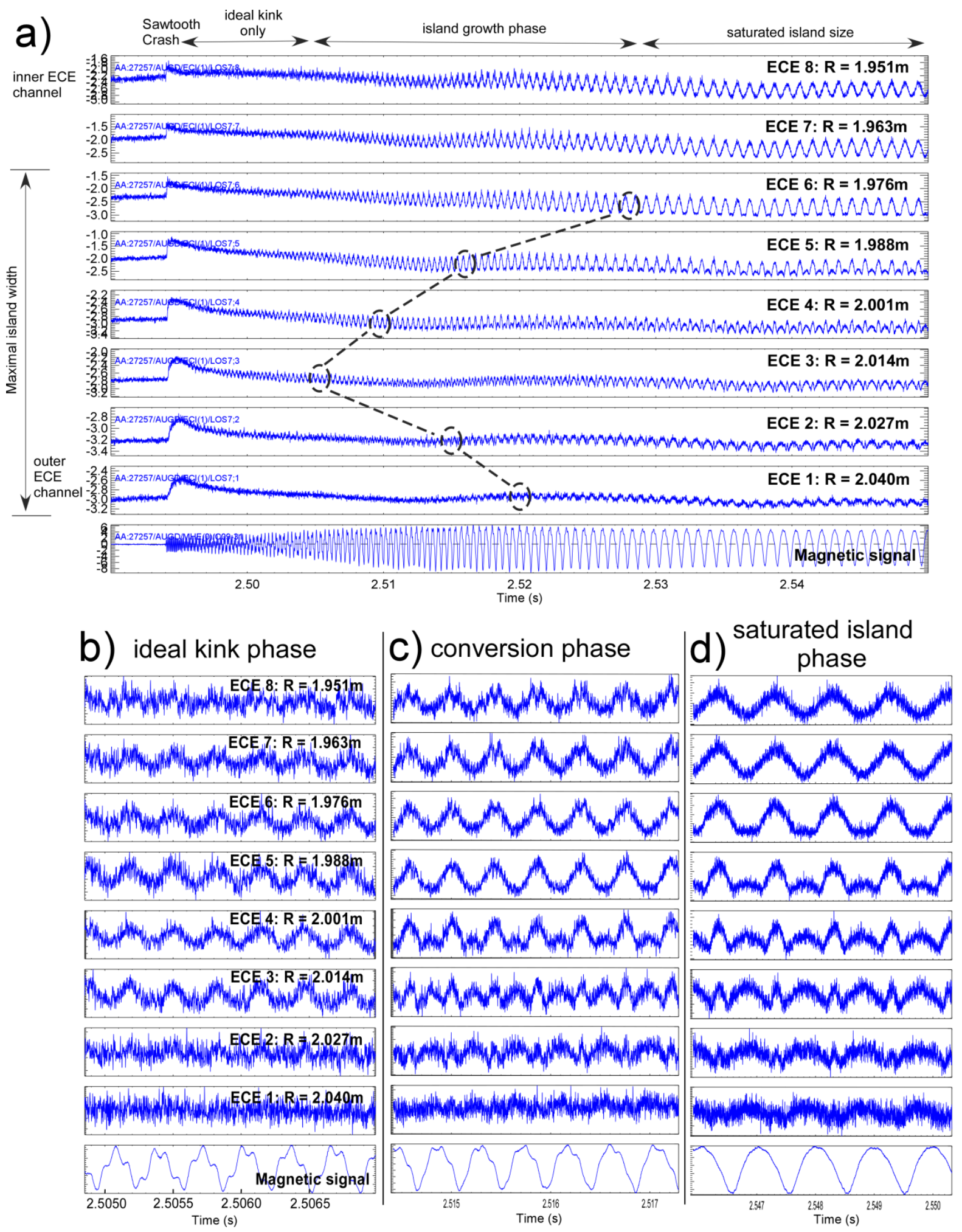

Figure 2. a) Direct identification of the transition from an ideal kink mode into a hot island in different ECE-Imaging channels for discharge \#27257 at $\mathbf{t = 2 . 5 s}$. The bottom time trace is the magnetic signal. b) The figure represents a small time window of figure (a) immediately after the sawtooth crash. All temperature perturbations are in phase, demonstrating the pure ideal character of the mode. $c$ ) Transition time is shown. d) Saturated island at the end of the mode evolution. 
In the following we will summarize the information about the total mode amplitude (ideal+tearing) from magnetic signals and the evolution of the island width from ECE. The total mode amplitude $A_{(2,1)}$ is extracted from the magnetic measurements as $A_{(2,1)} \sim \sqrt{b_{(2,1)}}$ [19]. Here, $b_{(2,1)}$ is the measured perturbation amplitude at the $(2,1)$ mode frequency filtered by Fourier. The mode amplitude $A_{(2,1)}$ is scaled to fit the island size from ECE at a later time point, when the saturated island size is reached and only the island component remains.

a)

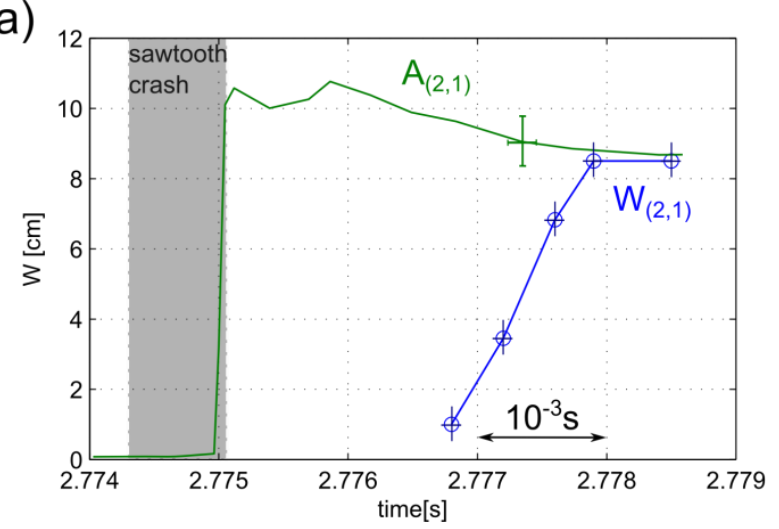

b)

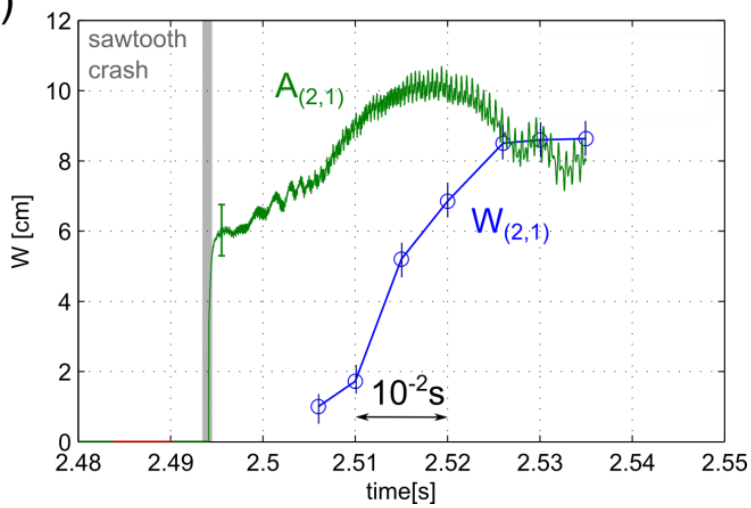

Figure 3. Comparison of the perturbation amplitude of the $(2,1)$ mode from magnetic signals, $A_{(2,1)}$, and the $(2,1)$ island width,$W_{(2,1)}$, from ECE measurements for two different cases: a) $\# 27257, t=2.77 \mathrm{~s}$, the same case as in figure $1 \mathrm{~b} ; \mathrm{b}) \# 27257, \mathrm{t}=\mathbf{2 . 5}$ s, corresponding to figure 2 . The shadow region indicates duration of the sawtooth crash as it is seen by central ECE channel. (Reproduced from [18], with the permission of AIP Publishing.)

Figures $3 \mathrm{a}$ and $\mathrm{b}$ show the evolution of the magnetic mode amplitude in comparison to the island size from ECE for the cases discussed above. The ideal mode is generated by the sawtooth crash directly after the crash. In figure $3 \mathrm{a}$, the mode keeps its ideal character for about $2 \cdot 10^{-3} s$ and only then transforms into an island structure within $10^{-3} s$. In figure $3 b$ the transition from ideal to tearing mode takes even longer. These time scales are much longer than the sawtooth crash time. The magnetic signal does not change during the mode conversion, indicating that the kink mode and the resulting tearing mode have identical helicity (figure $1 \mathrm{~b}$, bottom, between $2.776 \mathrm{~s}$ and $2.778 \mathrm{~s}$ ). The evolution of the mode frequency is also seen on spectrograms (figures 5 and 6).

The sawtooth crashes discussed above are not identical. The amplitude drops of the central ECE and SXR signals during the sawtooth crash for the fast conversion case (figure $3 a)$ are $32 \%$ and $40 \%$, respectively. The slow conversion case (figure $3 \mathrm{~b}$ ) has smaller drops (ECE 23\%, SXR 24\%) and the ideal mode amplitude immediately after the crash is smaller by almost a factor of two (figure 3). Kinetic profiles and the plasma rotation profiles are almost 
identical in these two cases, which excludes their influence on the mode conversion time (figure 4). The saturated island width, $W_{(2,1), s a t}=6.4 \mathrm{~cm}$, is also the same for these cases.
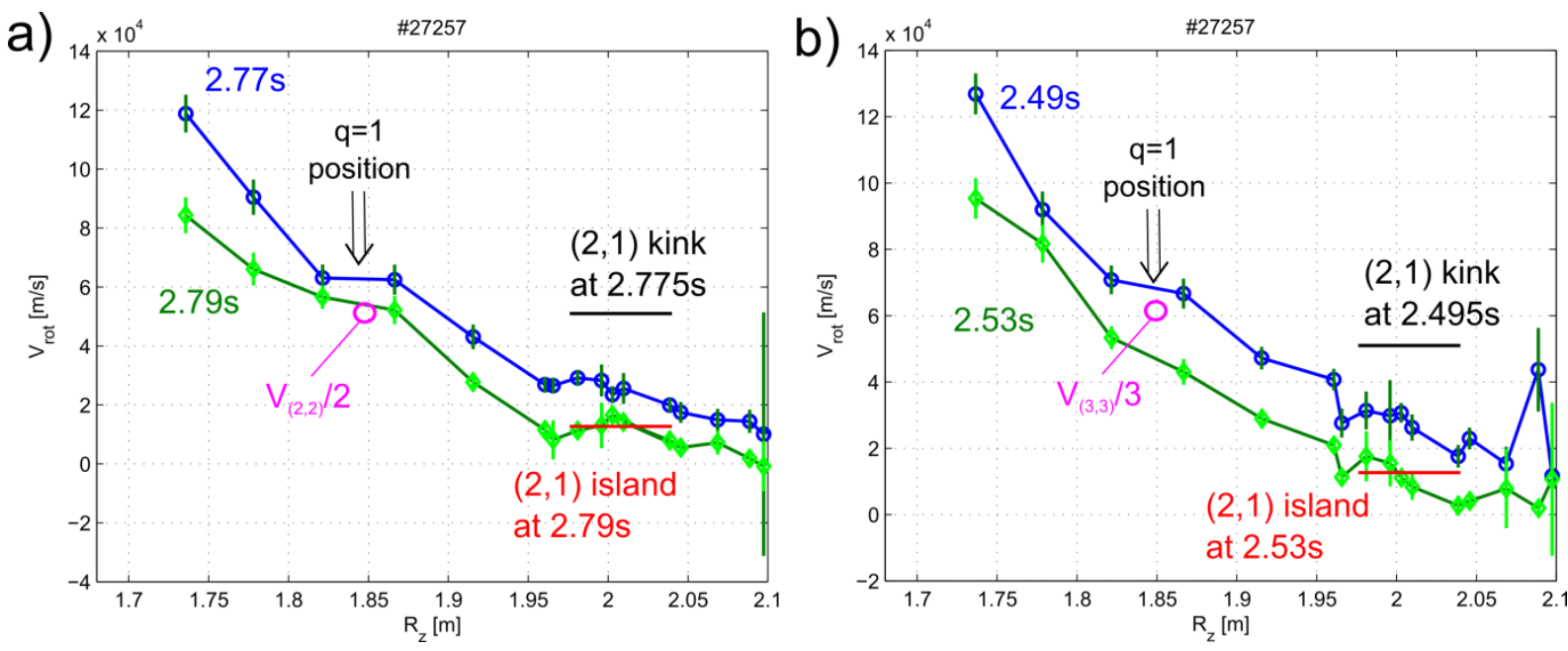

Figure 4. Toroidal plasma rotation before the crash and during the island saturation for the same cases as in figures 1, 2 and 3 (\#27257). Positions of the $q=1$ and $q=2$ resonant surfaces are indicated as well as the $(2,1)$ velocity during the kink and island phases. The radial extension of the red line corresponds to the saturated island size at the low field side. The velocity of the sawtooth precursor is also shown for $(2,2)$ and $(3,3)$ modes.

Thus, the difference in the mode conversion time is probably connected to the different amplitudes of the drive. At the same time, identical perturbation amplitudes could lead to completely different timescales if the plasma conditions are not identical. The Sweet-Parker time required for the island formation is about $0.1 s$ (see Appendix A.) and it is much longer than the observed mode conversion times. The single fluid assumption of the Sweet-Parker model is valid if the characteristic Larmor radius, $\rho_{s}$, is smaller than the width of the reconnection layer, which is the minimal length in the model. For the experimental plasma parameters at the $(2,1)$ resonant surface, the ion-sound Larmor radius, $\rho_{s}=c_{s} / \Omega_{i}=8.2$. $10^{-3} \mathrm{~m}$, by far exceeds the width of the Sweet-Parker layer, $\delta_{s p}=L / \sqrt{S}=1.2 \cdot 10^{-5} \mathrm{~m}$ here, $c_{S}=\sqrt{\gamma Z k_{B} T_{e} / m_{i}}$ is the sound speed, $\Omega_{i}$ is the ion gyrofrequency, $L$ is the length of the reconnection region and $S$ is the Lundquist number. Thus, the single fluid reconnection model is not an adequate description, as expected for collisionless fusion plasmas. A very important question in this respect is the origin of the ideal mode at the $\mathrm{q}=2$ resonant surface after the sawtooth crash and why it lasts so long after the crash. It is well known that ideal internal kink modes with $m \geq 2$ should be stable in tokamak plasmas at low $\beta$ [20,21]. A spectral analysis of standard ECE signals shows mode activity of the $(2,1)$ mode frequency simultaneously inside the $\mathrm{q}=1$ surface and at the $\mathrm{q}=2$ resonant surface right after the crash in 
both of the cases reported above (figure 5). Thus, the internal $(1,1)$ mode is toroidally coupled to the $(2,1)$ mode. It might thus be a surviving $(1,1)$ perturbation after the sawtooth crash that drives the reconnection at the $(2,1)$. This mode could either be a resistive $(1,1)$ kink mode or a long lasting ideal $(1,1)$ mode. Recent two-fluid modelling of sawtooth crashes demonstrated a long lasting ideal $(1,1)$ perturbation after a sawtooth crash [22]. In both variants, observed sawtooth crash indicates presence of $\mathrm{q}=1$ inside the plasma.
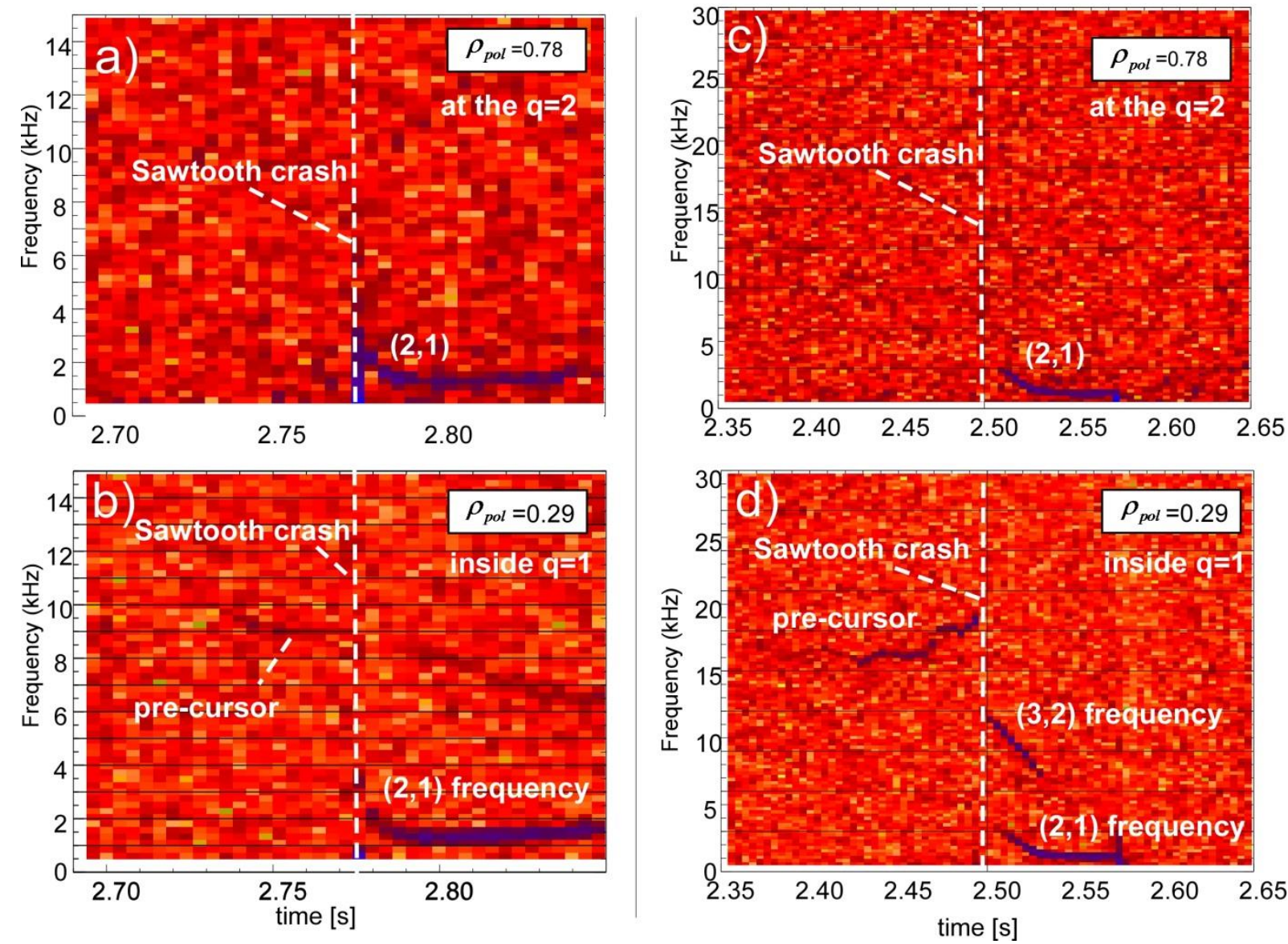

Figure 5. ECE spectrograms are shown for the same crashes as in figures 1 and 2 (\#27257). Channels at the resonant surface $q=2$ are shown in figures (a) and (c). Core channels (inside $q=1$ ) are shown in figures (b) and (d).

The overall MHD activity is more complex and is not restricted to the discussed $(2,1)$ mode. SXR and magnetics also show either a $(2,2)$ or a $(3,3)$ sawtooth pre-cursor and post-cursors in the two cases as seen in figure 6. 
a)

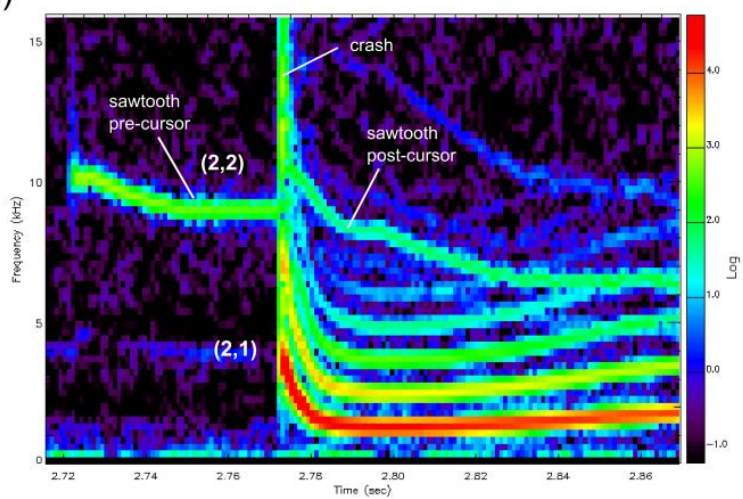

b)

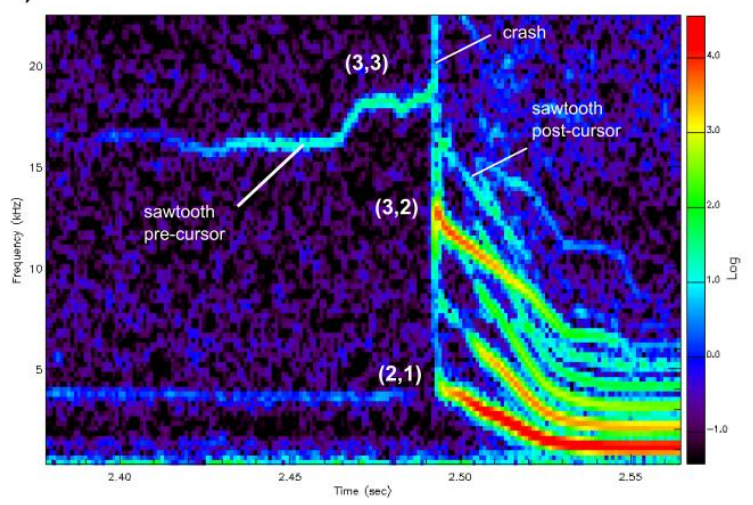

Figure 6. Spectrograms of magnetic signals (\#27257). a) Corresponding to the case in figure 1; b) For the same case as in figure 2.

All these observations suggest that the explanation for the existence of ideal $(2,1)$ kink modes is a strong $(1,1)$ perturbation after a sawtooth crash that drives the toroidally coupled $(2,1)$ mode. It is important to emphasise that our plasmas are intrinsically stable with respect to classical tearing mode $\left(\Delta^{\prime}<0\right)$, as expected for toroidal plasmas. Therefore, the island triggered by sawteeth at about $2.5 \mathrm{~s}$ decays to zero at $2.7 \mathrm{~s}$ in discharge \#27257. For the low beta plasmas investigated here, neoclassical drives are also negligible.

\section{Tearing mode seeding at high $\beta_{N}$}

In this section we describe tearing mode seeding in H-mode plasmas of ASDEX Upgrade. In this high beta regime, the plasma becomes more prone to NTM formation and already smaller perturbation amplitudes are able to trigger the mode. A typical case for an NTM trigger is shown in figure $7\left(\# 32456 ; I_{p}=0.8 M A ; B_{t}=2.5 T ; n_{e}=5.5 \cdot 10^{19} \mathrm{~m}^{-3} ; q_{95}=\right.$ 5.39; $\beta_{N}=2.61$ just before the mode onset). The high frequency mode $(\mathrm{n}=4$, determined from magnetic and SXR diagnostics) is not able to trigger an NTM alone. At some point this activity becomes coupled to the fishbone branch $(n=2$, detected in magnetic and SXR) and this event triggers the mode. The amplitude of the trigger is much weaker compare to the low beta cases in the previous section. 


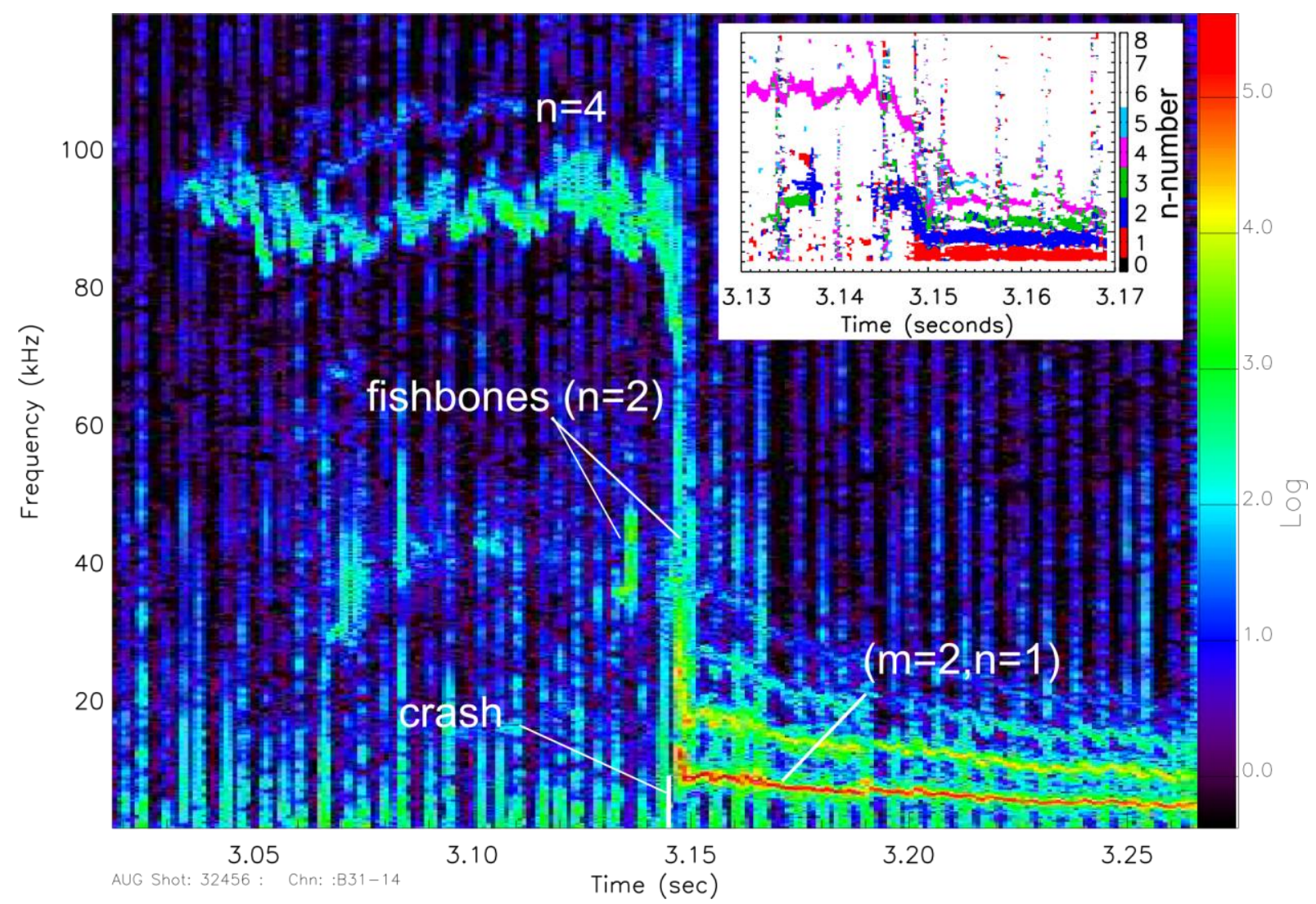

Figure 7. Spectrogram of the magnetic signal showing the trigger of a $(2,1)$ mode (\#32456). Inset: toroidal mode number analysis based on a set of magnetic coils.

Using the same technique as above, one can deduce the total mode amplitude from magnetic signals, and the island width from local ECE measurements. The result is shown in figure $8 \mathrm{a}$. In this case, the island grows quickly to $5 \mathrm{~cm}$ size. Further growth proceeds slowly and the full island size is reached after $70 \mathrm{~ms}$ only. The initial phase, represented in figure $8 \mathrm{~b}$, is very similar to the cases with sawteeth triggers reported in section 3. The fast growth of the ideal mode, $\mathrm{A}_{(2,1)}$, represents the kink mode formation at the rational surface directly after the crash. After a short delay, the island formation phase starts and takes about $1 \mathrm{~ms}$ to reach the size of about $5 \mathrm{~cm}$. Figure $8 \mathrm{c}$ shows the standard analysis of the phase between ECE channels, which also shows the ideal mode directly after the crash (the same phase for the first $1 \mathrm{~ms}$ ) [19]. The phase remains the same also during the time when a small island is already seen by a single ECE channel. The phase jump becomes obvious as soon as two ECE-channels see the island. This result is consistent with the previous findings. In this particular case, a tomographic reconstruction of the Soft X-ray radiation could give additional important information. It shows the appearance of toroidally coupled $(2,1)$ and $(1,1)$ modes directly after the crash. Due to the peaked radiation profile in this discharge, the amplitude of the $(1,1)$ displacement can be separated from the $(2,1)$ component. The detailed algorithm for the determination of the 
$(1,1)$ amplitude is described in references [23,24]. The $(1,1)$ amplitude rises together with the amplitude of the magnetic signal and represents the ideal mode in the plasma core. This mode is present for a sufficiently long time to allow island formation. In these discharges, the central safety factor is kept slightly above one and the excited $(1,1)$ mode might be driven by the pressure gradient in the low shear region (quasi-interachange [25], infernal mode [26] or long living mode [27]). Such a perturbation can also be excited with external B-coil perturbations at high normalized beta [28]. (Equilibrium plasma profiles are shown in figure 15 for this case.)
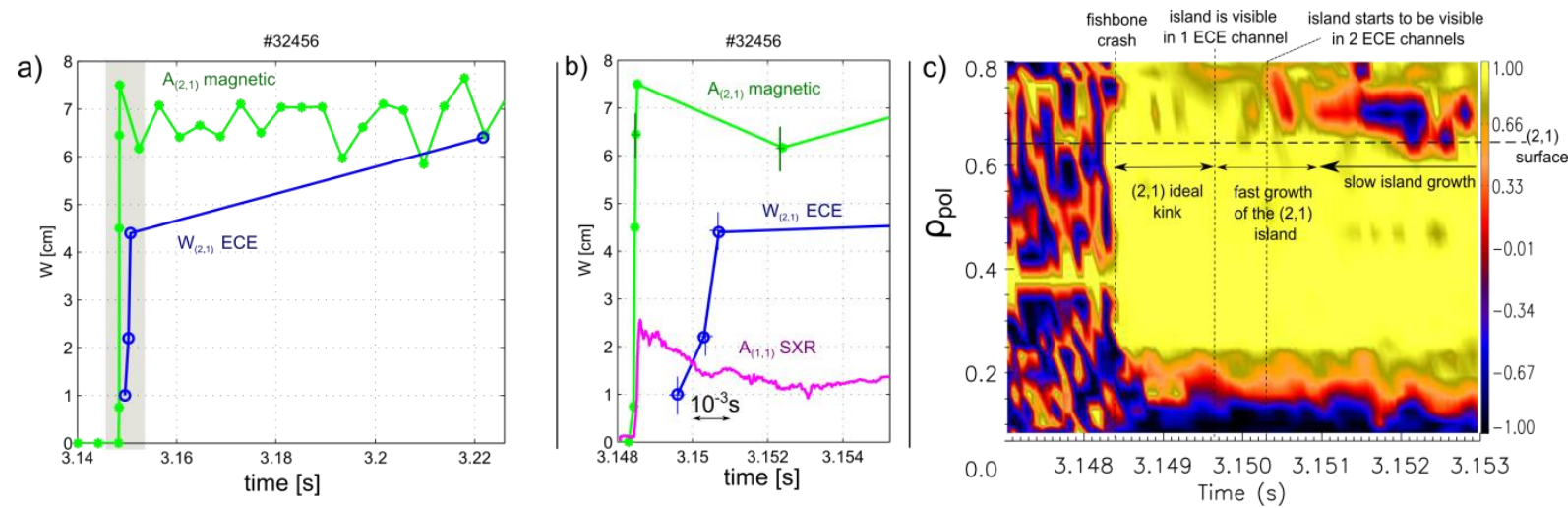

Figure 8. (a) Comparison of the perturbation amplitude of the $(2,1)$ mode from magnetic signals, $A_{(2,1)}$, and $(2,1)$ island width,$W_{(2,1)}$, from ECE; (b) The figure shows the fast evolution phase, which is marked as a grey region in figure (a). $A_{(1,1)}$ SXR is the core displacement obtained from SXR tomography using the algorithm described in ref $[23,24]$. The error bars of the measurements are shown; (c) Phase crosscorrelation of the ECE signals.

Changes in temperature and rotation profiles after mode onset are shown in figure 9. The pure ideal phase of the mode is shown as a white line in figure 9a which converts into a red curve as far as the island starts to grow. The observed drops in temperature (11\%) and SXR radiation $(5 \%)$ are significantly smaller compared to the low beta cases discussed in the previous section. 

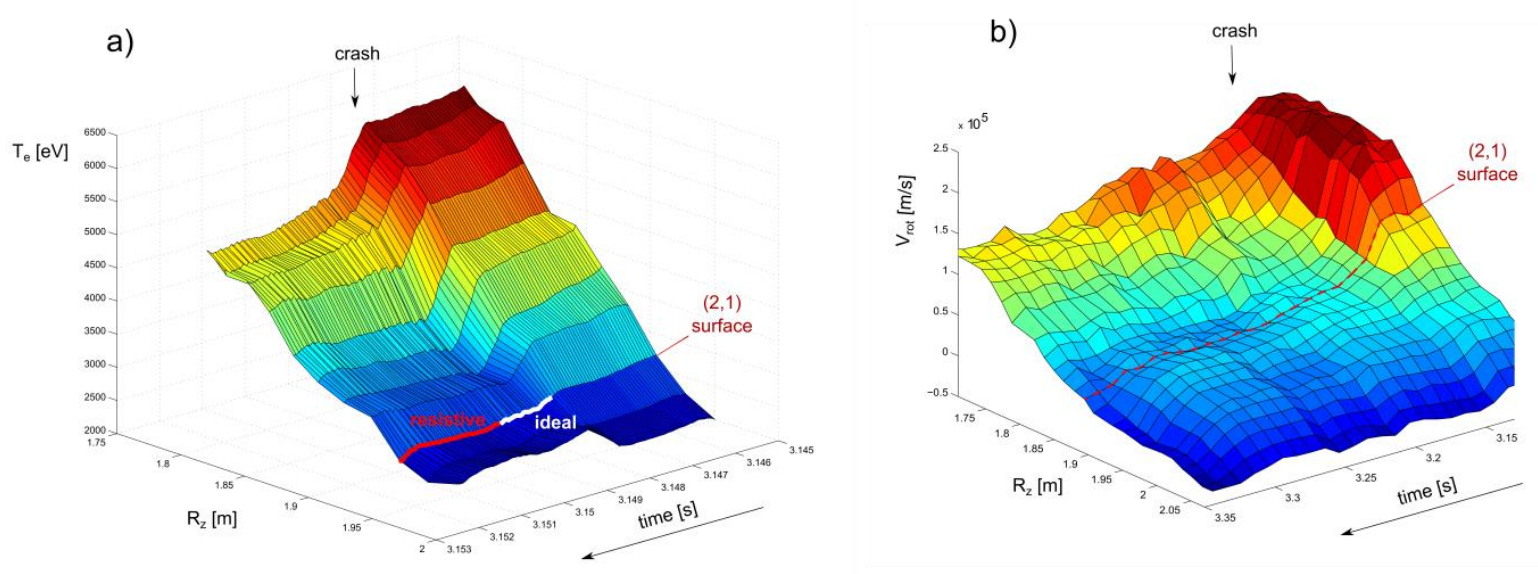

Figure 9. Temperature (a) and rotation (b) profiles for the same case as in figure 7 (\#32456, $t=3.148 s)$. The resonant surface $q=2$ is shown as a line in both figures.

The mode rotation frequency is close to the plasma rotation at the $q=2$ surface as shown in figure 10. This is also true for the initial ideal $(2,1)$ kink mode and is different compared to the cases discussed in section 3 .

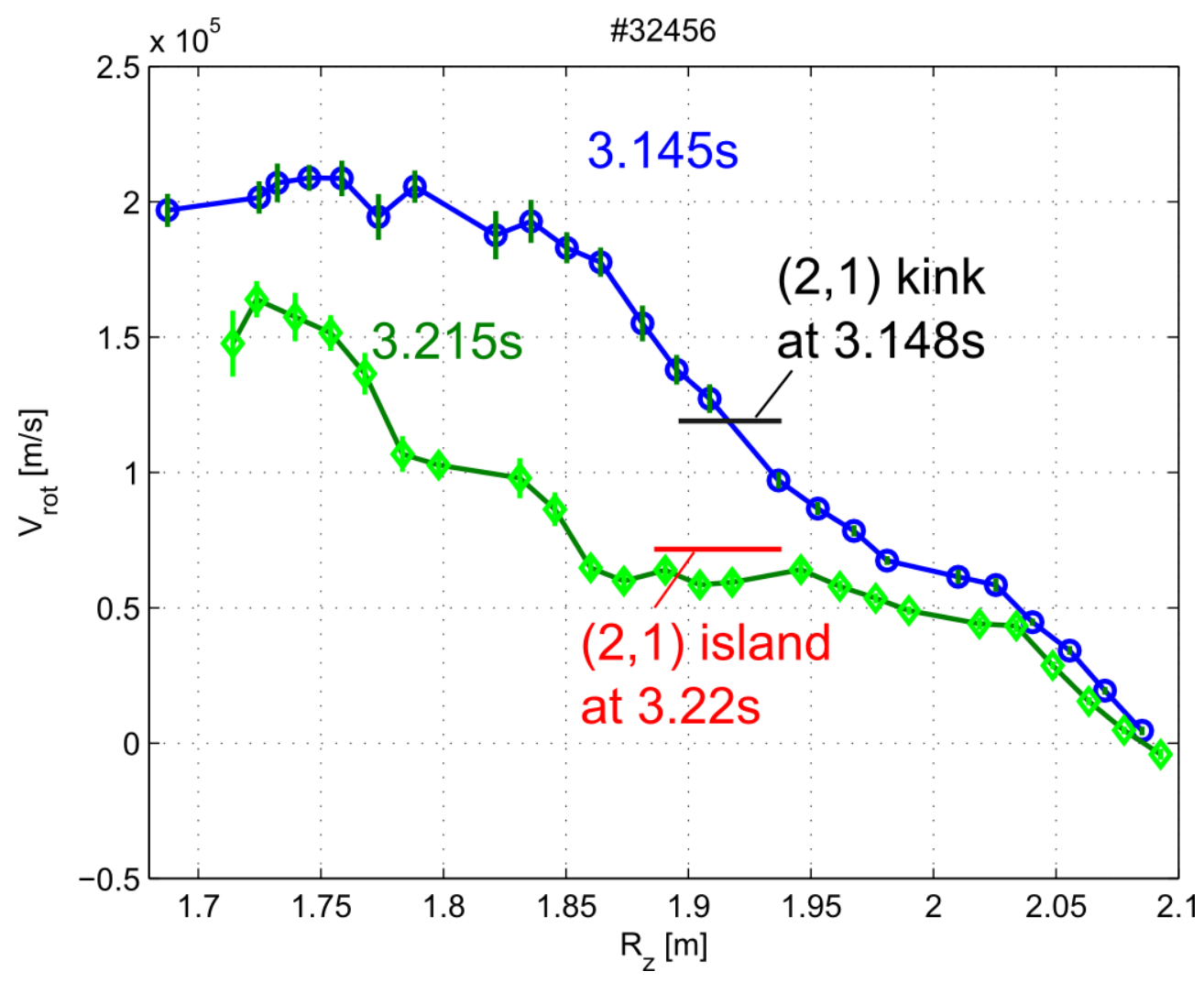

Figure 10. Toroidal plasma rotation profiles just before the crash $(t=3.145 \mathrm{~s})$ and after the island formation $(t=3.22 s)$ are shown for the same case as in figures 6,7 and 8 . The position and size of the $(2,1)$ island are indicated in red. The position and rotation velocity of the ideal $(2,1)$ kink mode are shown in black.

Another example of NTM seeding at high beta is considerably different and shown in figure $11\left(\# 32354 ; I_{p}=0.8 M A ; B_{t}=2.5 T ; n_{e}=5.2 \cdot 10^{19} m^{-3} ; q_{95}=5.47 ; \beta_{N}=2.56\right.$ just before 
the mode onset). In this case, the safety factor profile has two $q=2$ surfaces (figure 15) and two modes with $(2,1)$ helicity coexist in the plasma at these surfaces. The modes are coupled and the resulting structure is called either double kink or double tearing mode depending on the mode structure. In this situation, magnetic perturbations represent the amplitude of the outer mode. 


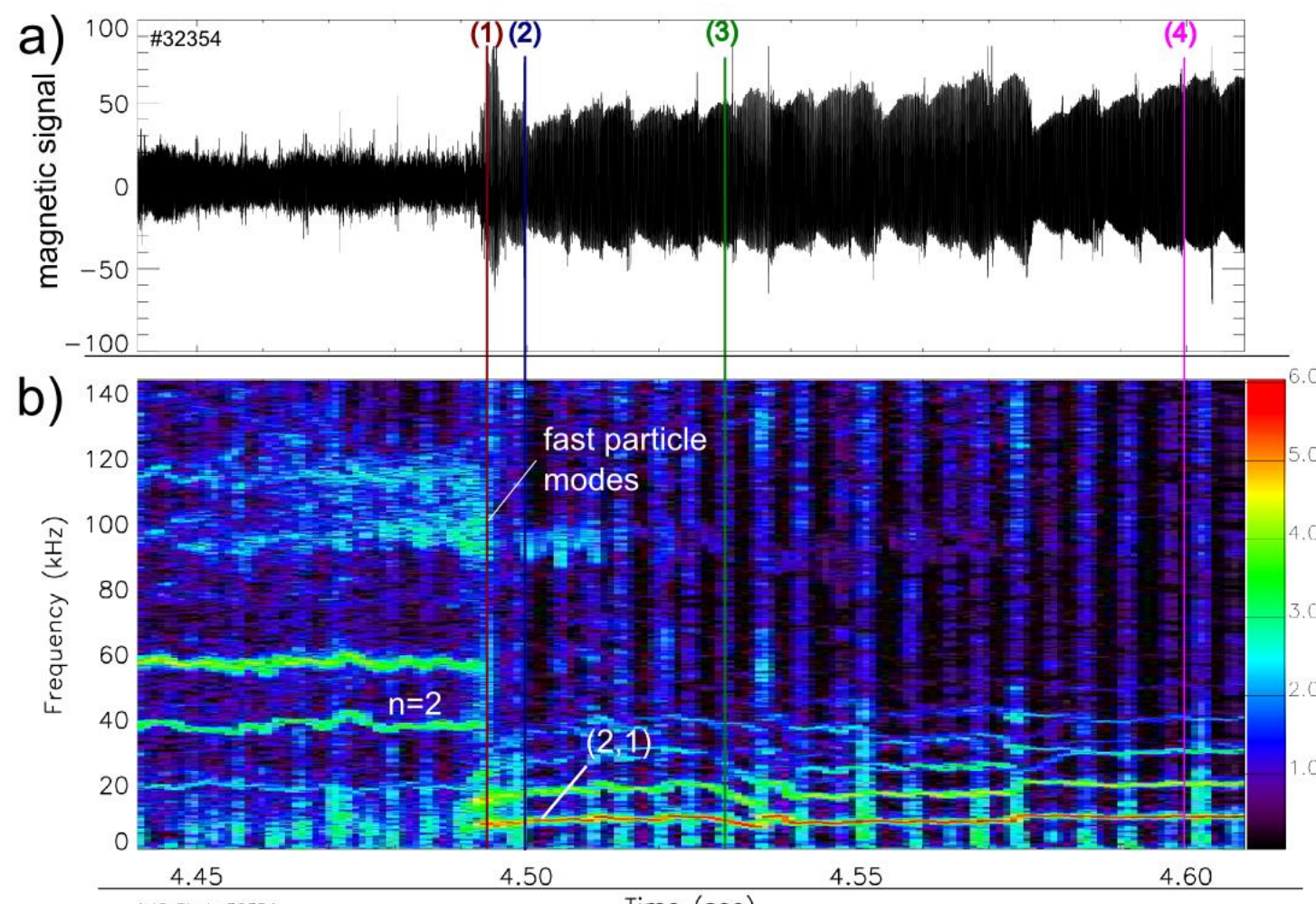

c)
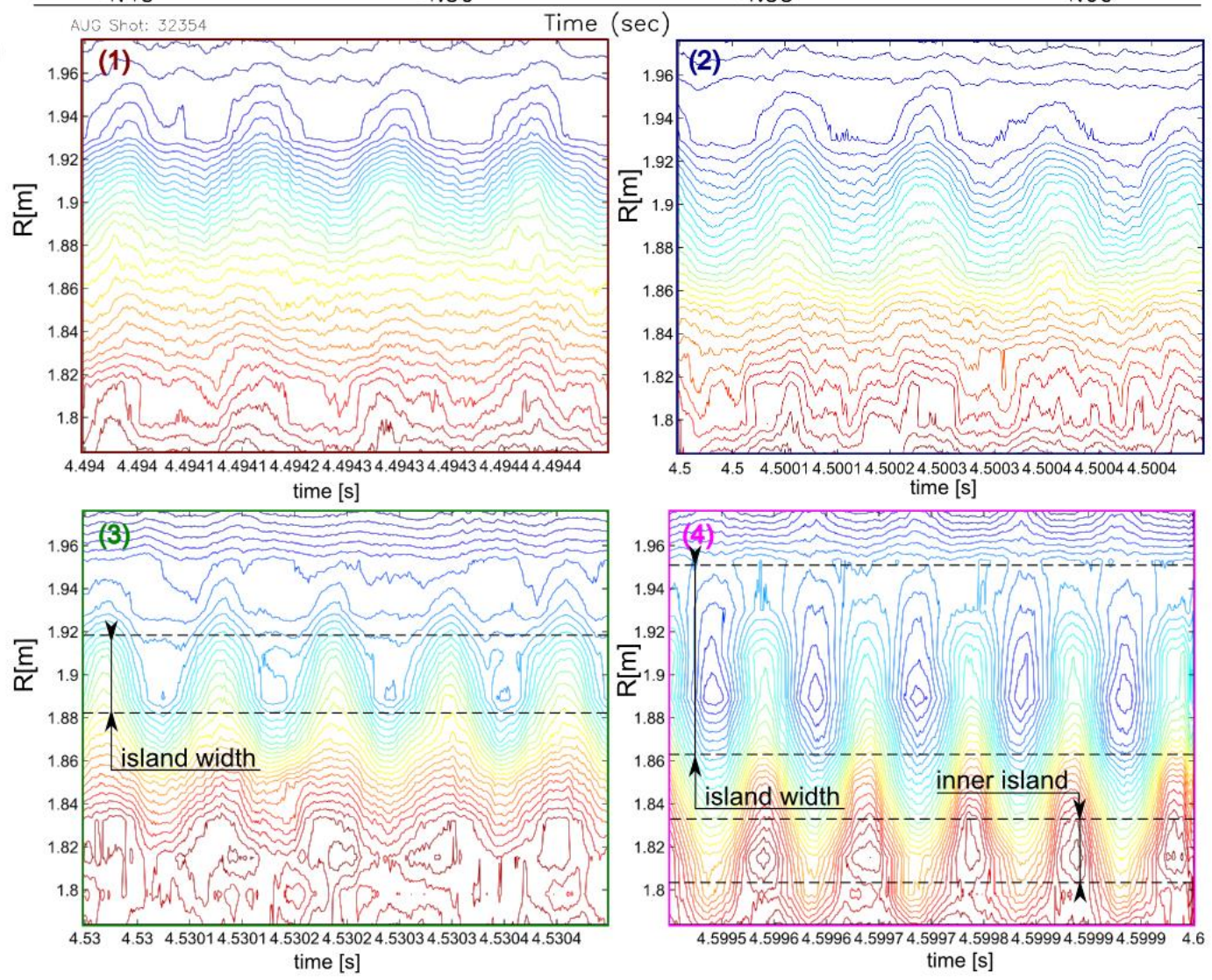

Figure 11. (a) Magnetic signal for discharge \#32354; (b) Spectrogram of the magnetic signal (a); (c) contour plots of the temperature isotherms from the standard ECE diagnostic for four different time points indicated in figures (a) and (b) by vertical lines 1-4. 
The pre-crash weak core MHD activity with $n=1,2,3$ is located inside the $\rho_{\text {pol }} \approx 0.6$ region (determined from ECE and SXR diagnostics). The exact details are hardly extractable due to the small mode amplitude. This mode activity is accompanied by a fast frequency mode at about $100 \mathrm{kHz}$. The seeding process starts with the ELM at $\mathrm{t}=4.491 \mathrm{~s}$. The following "fishbone like" sweep of the $\mathrm{n}=2$ mode at $\mathrm{t}=4.492$ s lead to formation of the large amplitude mode at $\mathrm{q}=2$ surface. As a result, only $(2,1)$ kink mode remains after the crash. The ECE signals show a constant phase directly after the mode formation and a clear phase jump due to an island at a later time. This can also be seen directly on the ECE contour plots. The first (1) and the second (2) contour plots of ECE temperature signals in figure 11c demonstrate clear kink mode activity $\left(R_{z} \approx 1.92 \mathrm{~m}\right)$ which is accompanied by the inner kink mode $\left(R_{z} \approx 1.82 \mathrm{~m}\right)$. At a later time, the outer island formation is clearly seen in figure $11 \mathrm{c}(3)(\mathrm{t}=4.53 \mathrm{~s})$. The last contour plot in figure 11c(4) shows two islands of the double tearing mode. The total mode amplitude and the island size evolution at the $\mathrm{q}=2$ surface were determined in the same way as discussed in section 2 (figure 12). The island formation in this case takes even longer compared to the previous example. At the same time, the amplitude drops in ECE and SXR are similar to the previous high beta case (\#32456) within the error bars of the measurements. A comparison of figures $8 \mathrm{~b}$ and 12 demonstrates differences by at least one order of magnitude in the timescales as well as a different dynamic of the island formation. The seeding process can thus not be described only by the size of the sawtooth crash itself. Both, the plasma parameters and the kind of mode activity right after the crash are relevant as well. 


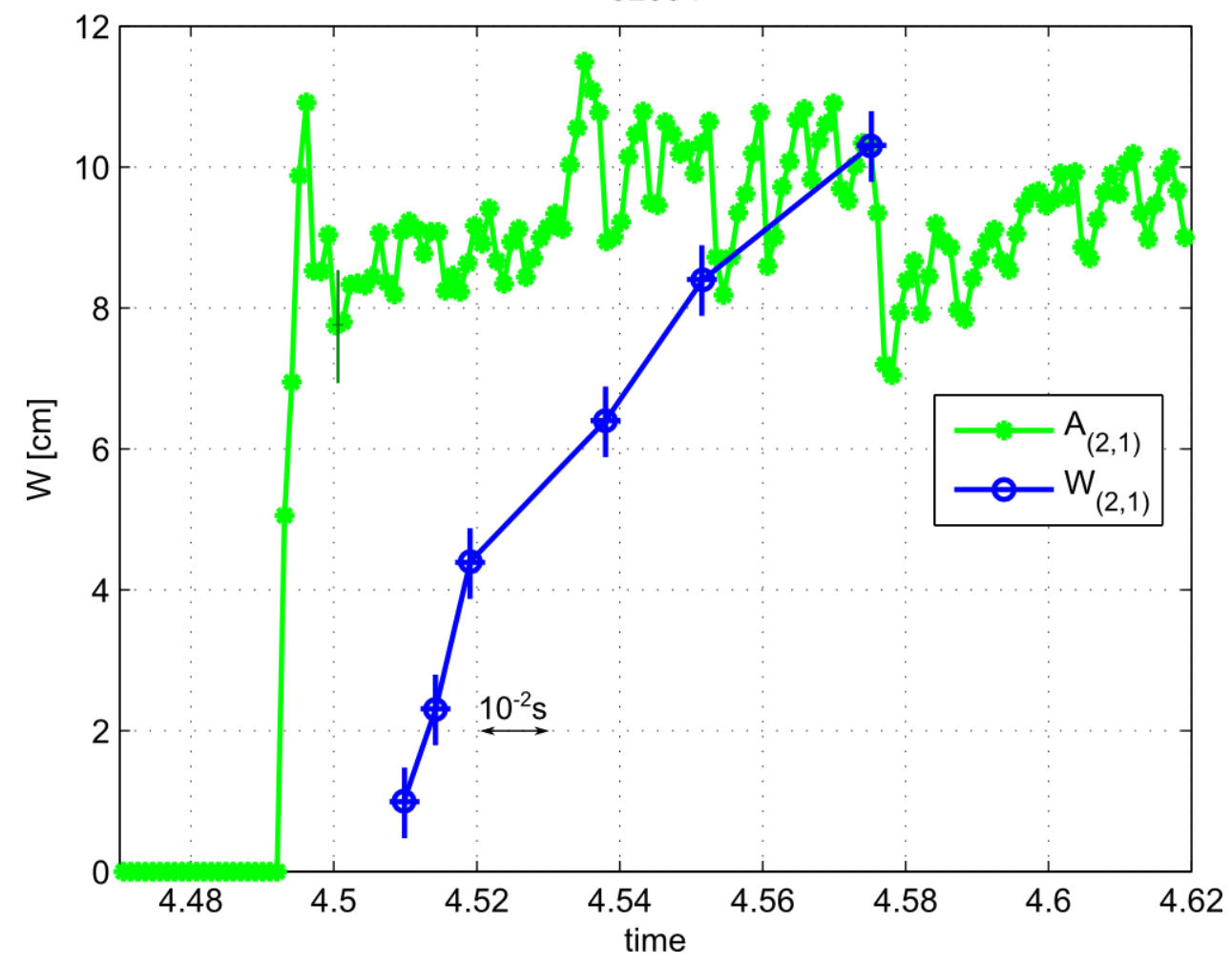

Figure 12. Comparison of the perturbation amplitude of the outer $(2,1)$ mode from magnetic signals, $A_{(2,1)}$, and outer $(2,1)$ island width,$W_{(2,1)}$, from ECE for the same case as in figure 9 (\#32354).

The same case was investigated with SXR tomography. Experimental measurements of magnetic islands show that the inner part of the islands is significantly broader than the outer part [29]. Thus, the SXR fluctuation signal is dominated by the inner island region which is colder at the O-point and hotter in the X-point compared to the mean signal. The number of the hot/cold regions corresponds to the poloidal mode number, $m$. Figure 13 clearly shows two modes with $m=2$ helicity which are coupled in the opposite phase. This coupling pattern is the characteristic for double kink/tearing modes [30]. These observations suggest that the $q$-profile is reversed in this case and slightly higher in the core compared to previous examples. 

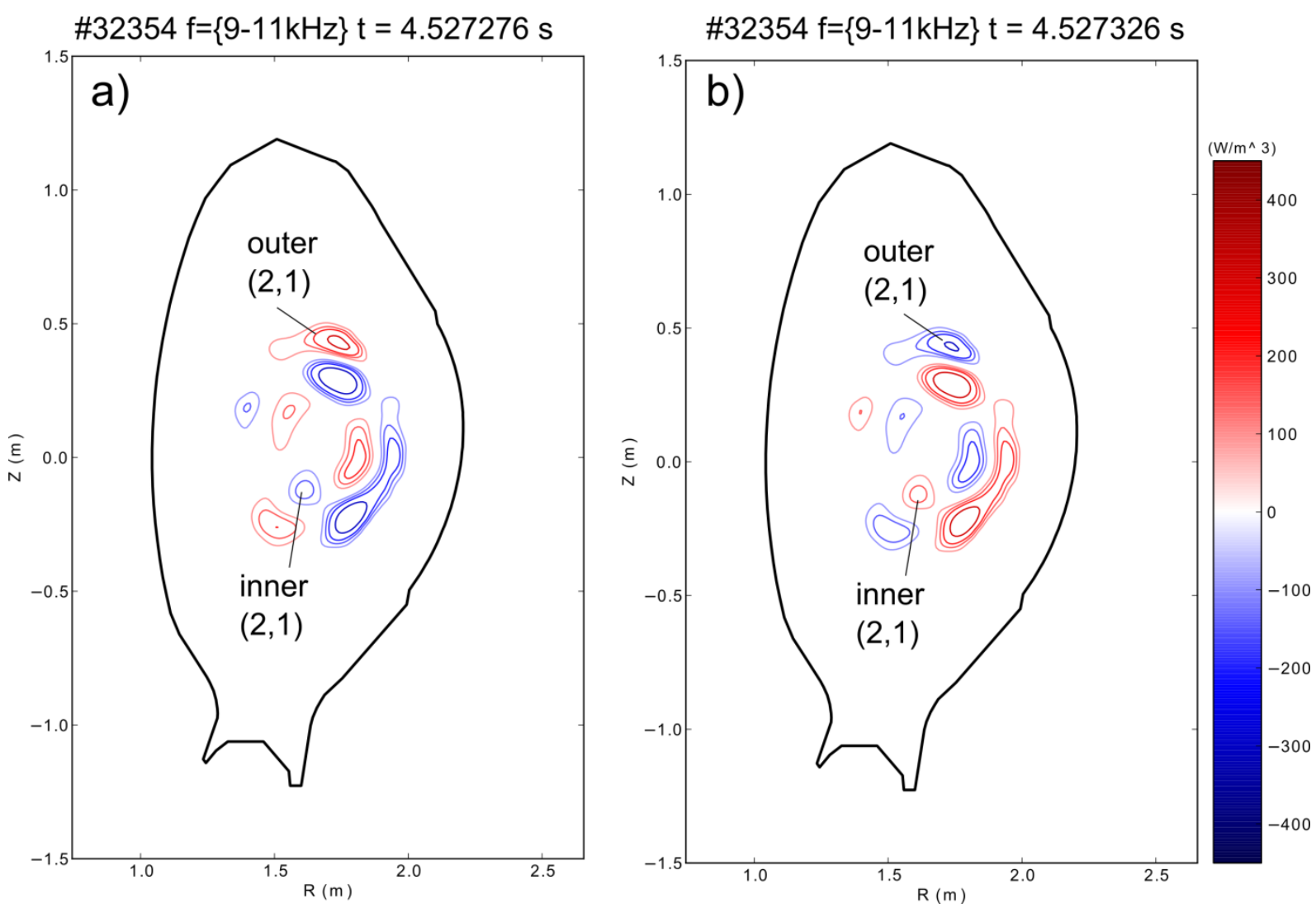

Figure 13. Tomographic reconstruction of Soft $X$-ray perturbations at the mode frequency for two opposite phases of $(2,1)$ modes. A double $(2,1)$ mode is clearly visible in both figures. (The mean SXR emissivity is subtracted to make the mode visible.)

The toroidal plasma rotation just before the crash $(\mathrm{t}=4.45 \mathrm{~s})$ and after the island formation $(\mathrm{t}=4.59 \mathrm{~s})$ is shown in figure 14. The mode frequency is constant in this example, and it matches closely the plasma rotation. The positions of the modes and their saturated widths are shown in red in figure 14. 


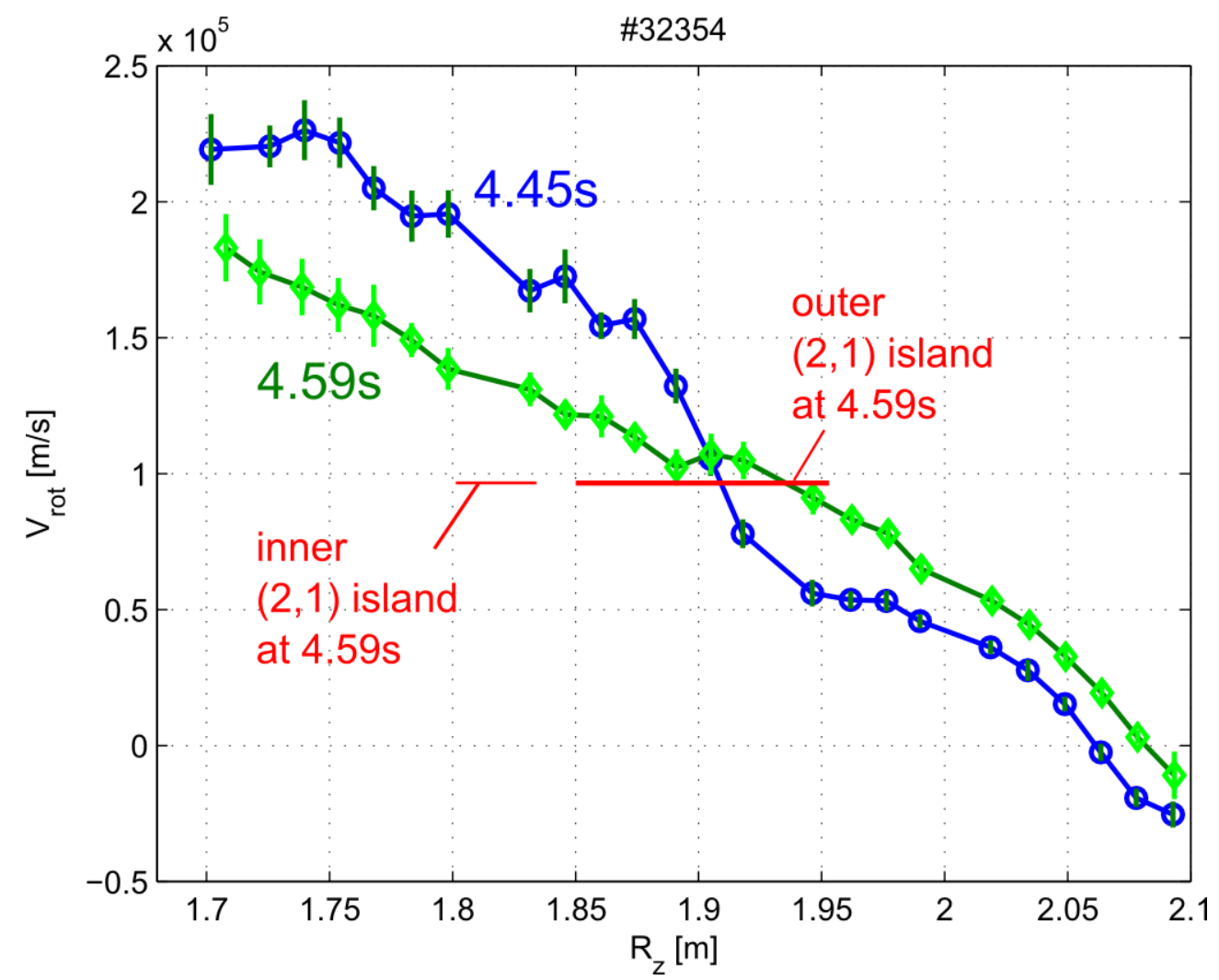

Figure 14. Toroidal plasma rotation just before the crash $(t=4.45 \mathrm{~s})$ and after the island formation $(t=4.59 \mathrm{~s})$ are shown for the same cases as in figures 11,12 and 13 . Positions and sizes of the $(2,1)$ islands are indicated in red.

Plasma profiles reconstructed by CLISTE code with MHD constraints [31] for the high beta cases discussed in this section are shown in figure 15. In both cases, the reconstructed profiles just before the crash events are plotted. The first case (\#32456) has safety factor profile slightly above one and this allows to have an internal ideal $(1,1)$ component which is detected by SXR tomography and shown in figure $8 \mathrm{~b}$. The central safety factor behavior is similar to the improved H-mode scenario. The second case (\#32354) has a slightly reversed shear region and is more close to the advanced tokamak scenario aimed on steady state tokamak operation. This scenario has large fraction of the non-inductively driven bootstrap current, which lead to reversed safety factor profiles and appearance of the resonant surfaces with identical helicities inside the plasma. Indeed, the double kink/tearing mode was observed in this case in the experiment (see figures 11 and 13). One of the main dangers of this scenario is global kink mode which converts into resistive wall mode. This mode is expected to be unstable above the "no-wall" limit. Up to this limit, kink modes should be stable even without the stabilizing influence of the external conducting wall. Stability calculations with CAS3D/STARWALL [32] codes show that the "no-wall" limit is at 
$\beta_{N, n o-\text { wall,thermal }}=2.4$ for this plasma. The experimental value ${ }^{1}$ achieved in the discharge is $\beta_{N, \text { exp,thermal }}=2.02\left(\beta_{N, \text { tot,exp }}=2.56\right)$. This indicates that the plasma is slightly below the "no-wall" limit and should be stable with respect to kink modes.
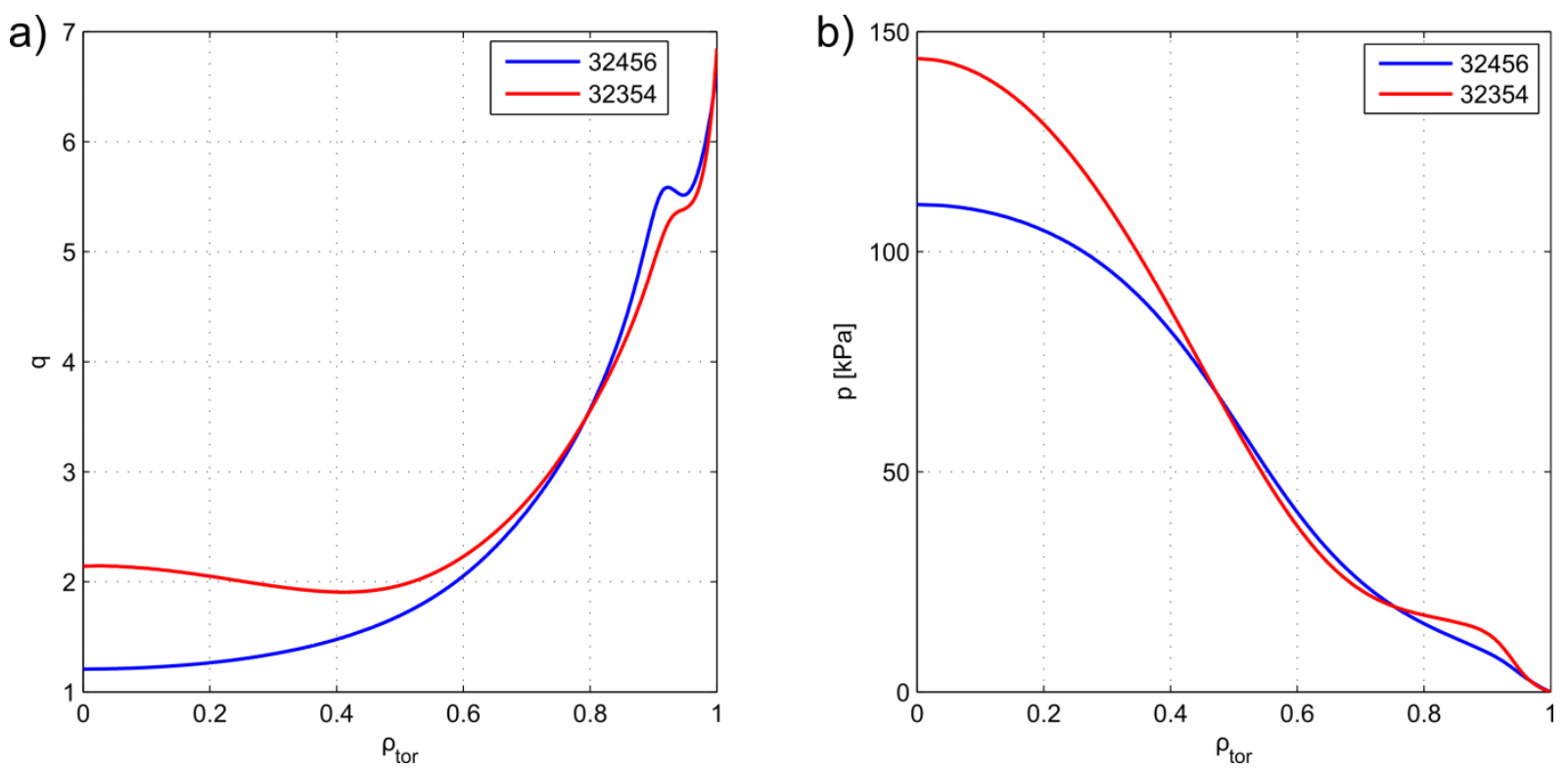

Figure 15. Equilibrium plasma profiles from equilibrium reconstruction with CLISTE [31] just before the crash events for two cases discussed in this section: a) safety factor profiles; b) pressure profiles.

\section{Conclusions and discussion}

Forced magnetic reconnection during the tearing mode seeding by MHD events is investigated in the paper. Complex analysis of the MHD information from SXR, magnetics and ECE during the seeding process shows that the simplified picture, which assumes the formation of a big island during the crash, has to be revised. A large tearing mode requires time for its formation and has not been observed during or immediately after the crash. The dominant mode after the crash is an ideal kink mode. This conclusion is based on local ECE measurements from two independent ECE diagnostics (standard ECE and ECE Imaging). The applied analysis of the ECE time traces, described in section 2, is verified by the standard analysis for the ECE phase jump position (figure 8c) and contour plot analysis of ECE data (figure 11c). These widely used methods are not as sensitive as our main method, but their results are in agreement with our results. The analysis presented is able to detect islands down to a size of $1.5-2 \mathrm{~cm}$ and would unavoidably detect a big island formation during the crash. It is important to emphasise that no conclusions regarding the existence of a smaller island directly after the crash can be given. As mentioned before, small islands have no influence on

${ }^{1}$ These $\beta_{N, \text { thermal }}$ assume only thermal particles for proper comparison. In all other places in the paper, total normalized beta is used. It includes also fast particle pressure. 
the temperature profile. In that respect, the lower limit of our detection method is set by the physical effect of the island on the temperature profile, and not by the diagnostic resolution.

The main conclusions from our studies of the seeding process are the following:

(1) An internal crash (sawtooth/fishbone) creates a kink mode at the resonant surface, where the island will be formed.

(2) This kink has the same helicity as the subsequent island. In our cases, this was always a $(2,1)$ mode. The kink is coupled to the internal post-crash activity.

(3) This $(2,1)$ kink mode converts into a $(2,1)$ tearing mode on a longer timescale $\left(10^{-3}-\right.$ $10^{-2} s$ ) compared to the crash time and moreover it is delayed with respect to the crash. At the same time, this conversion is much faster compared to the single fluid Sweet-Parker reconnection time (0.1s, see Appendix A). Thus, two fluid effects are important, which is also confirmed by comparison of the characteristic Larmor radius, $\rho_{s}$, with the width of the reconnection layer, $\delta_{s p}$ (see section 3 ).

(4) The island formation time does not strongly depend on the plasma conditions. The time scale ranges are similar for low $\beta_{N}$ (L-mode) and high $\beta_{N}(\mathrm{H}$-mode) cases. Thus, the forced reconnection during the seeding process is similar for classical and neoclassical tearing modes. The driving force due to the bootstrap current is only important for the further island evolution. In low-beta plasmas where the neoclassical drive is not important, the seeded tearing modes decay, indicating a negative $\Delta^{\prime}$ in the Rutherford equation [7].

(5) If the plasma conditions (rotation profiles, current profiles, position of the resonant surfaces, etc.) are different, the same amplitude of the perturbation can result in completely different conversion times. This is demonstrated by high $\beta_{N}$ examples.

(6) In our example, for otherwise similar plasma conditions, the higher perturbation amplitude led to the faster conversion time.

(7) The perturbation amplitude required to seed a tearing modes at high $\beta_{N}$ is much lower compared to the low $\beta_{N}$ case. (This was to be expected as high beta plasmas are more unstable with respect to both ideal modes and tearing modes.)

All these points agree with first numerical simulations with two-fluid non-linear MHD code [18]. "The physical mechanism in our case is: (i) slowdown of the plasma rotation; (ii) optimal rotation for best flux penetration accompanied by fast island growth; (iii) non-linear 
evolution." The important result of the calculations is that the conversion time (for the plasma conditions studied) is always longer compared to the typical sawtooth crash time.

The main question which is asked about NTM seeding is: "How to avoid tearing mode seeding, in particular at high $\beta_{N}$ ?". The answer depends strongly on the details of the seeding process. The island is not yet formed at this time. The main mode is the kink and the main question is how to stabilize this kink and why this kink is unstable. It is clear from simple MHD analysis that isolated internal kink has to be stable at low $\beta$ but our $(2,1)$ kink mode after the crash is not isolated. It is coupled to internal post-crash modes, which is seen on ECE and SXR. It could also be coupled to external kink modes, which would influence the mode stability. Thus, we have a situation similar to the advanced tokamak scenario, where the mode eigenfunction is broad and many poloidal mode numbers are coupled. In the case of the sawtooth crash, one can argue that the $(1,1)$ mode lives long enough after the crash to drive reconnection at $\mathrm{q}=2$ surface. Another variant of the $(2,1)$ formation has to be considered for high beta cases where no $\mathrm{q}=1$ is present. In this situation, the ideal MHD equilibrium can have several possible solutions and the axisymmetric solution has a minimum energy comparable to a solution with an ideal mode inside the plasma [33,34]. If the perturbations due to a fishbone crash are large enough, the plasma jumps from an axisymmetric state into a state with an ideal mode. This mode could live sufficiently long which gives time for island formation. It is possible that the answer on our question is not located at the NTM resonant surface and a broader view is required to solve the NTM seeding problem.

\section{Acknowledgement}

This work was partially funded by the Max-Planck/Princeton Center for Plasma Physics.

This work has been carried out within the framework of the EUROfusion Consortium and has received funding from the Euratom research and training programme 2014-2018 under grant agreement No 633053. The views and opinions expressed herein do not necessarily reflect those of the European Commission.

Valentin Igochine thanks Phillip Lauber for important comments and many discussions on fast particle physics. 


\section{Appendix A. Calculation of the Sweet-Parker reconnection time for $(2,1)$ mode}

Derivation of the Sweet-Parker reconnection time for tearing mode is based on the two main points:

(1) Maximal outflow velocity is well defined and limited in this model.

(2) One has to push enough plasma through the X-point region to fill the island volume.

The maximal possible outflow in the Sweet-Parker model is limited by Alfven velocity:

$v_{\text {out }}=v_{\text {Alfven }}=\hat{B} / \sqrt{\mu \rho}$, where $\hat{B}$ is reconnected helical magnetic field which is a part of poloidal magnetic field at the $\mathrm{q}=2$ surface.

$$
\begin{gathered}
q \approx \frac{r_{q=2}}{R_{0}} \frac{B_{\text {tor }}}{B_{\theta}}=2 \\
\hat{B}=B_{\theta}\left(1-\frac{q(r)}{q_{\text {res }}}\right) \approx 0.1 \cdot B_{\theta}=0.1 \frac{r_{q=2}}{R_{0}} \frac{B_{\text {tor }}}{2} \approx 0.028[T]
\end{gathered}
$$

The reconnected helical field, $\hat{B}$, is always only a fraction of the poloidal magnetic field.

The result Alfven velocity is:

$$
v_{\text {Alfven }}=\frac{\hat{B}}{\sqrt{\mu \rho}}=\frac{0.028 T}{\sqrt{4 \pi \cdot 10^{-7} \cdot 3 \cdot 10^{19} \cdot 3.3435 \cdot 10^{-27}}}=7.8 \cdot 10^{4}[\mathrm{~m} / \mathrm{s}]
$$

Standard relation between inflow and outflow in Sweet-Parker model is [35]:

$$
\frac{v_{\text {in }}}{v_{\text {out }}}=\frac{1}{\sqrt{S}}
$$

here $S$ is the Lundquist number and $v_{\text {out }} \equiv v_{\text {Alfven }}$. During the reconnection process one has to fill the volume of the island with the plasma: $V_{i s l} \approx \frac{1}{2} W_{i s l} \cdot L_{i s l}$, where $W_{i s l}$ is the island width and $L_{i s l}$ is the island length $\left(L_{i s l}=2 \pi r_{r e s} / m \approx 1.2[m]\right)$. The volume is filled with inflow velocity $v_{\text {in }}$ through the channel width $l \approx 0.02 m: V_{i s l}=v_{i n} \cdot \tau_{s p} \cdot l$. This leads to the reconnection time estimation:

$$
\tau_{s p}=\frac{W_{i s l} \cdot L_{i s l}}{2 \cdot v_{\text {in }} \cdot l}=\frac{W_{i s l} \cdot L_{i s l} \cdot \sqrt{S}}{2 \cdot l \cdot v_{\text {Alfven }}} \approx \frac{0.06 \cdot 1.2 \cdot \sqrt{3.6 \cdot 10^{7}}}{2 \cdot 0.02 \cdot 7.8 \cdot 10^{4}}=0.14[s] .
$$

\section{References}

\footnotetext{
${ }^{1}$ M.Yamada, R.Kulsrud, H.Ji, Rev.Mod.Phys. (2010) 603

${ }^{2}$ H.P.Furth, J.Killeen, M.N.Rosenbluth, Phys. Fluids, (1973), 1054

${ }^{3}$ S. Fietz, et.al., Pl. Phys. Contr. Fus. 55 (2013) 085010
} 
${ }^{4}$ A.Gude, S.Günter, S.Sesnic, Nuclear Fusion, Vol.39 (1999), p. 127

${ }^{5}$ R.J.Buttery et.al. Pl. Phys. Contr. Fus. 42 (2000) B61-B73

${ }^{6}$ M.Maraschek, Nucl. Fusion., 52 (2012) 074007

${ }^{7}$ M. Maraschek, Chapter 8 in V. Igochine (ed.) "Active Control of Magneto-hydrodynamic Instabilities in Hot Plasmas" (Springer Series on Atomic, Optical, and Plasma Physics, Vol 83, 2015)

${ }^{8}$ F.M.deBock, et.al., Nucl. Fus.48 (2008) 015007

${ }^{9}$ O. Sauter, C. Angioni, Z.R. Lin-Liu, Phys. Plasmas 6 (1999) 2834

${ }^{10}$ O. Sauter, et.al., Pl. Phys. Contr. Fus. 44 (2002) 1999

${ }^{11}$ T.C.Hender, et.al., Nucl.Fusion 47 (2007) S128

${ }^{12}$ O. Sauter, et.al., Phys. Rev. Lett. 88 (2002) 105001

${ }^{13}$ G.P.Canal, et. al., Nucl. Fusion 53 (2013) 113026

${ }^{14}$ P. Buratti et.al. Nucl. Fusion 52 (2012) 023006

${ }^{15}$ V. Igochine, A. Gude, M.Maraschek, "Hotlink based Soft X-ray Diagnostic on ASDEX Upgrade", report IPP 1/338, Mai 2010, 2 available http://pubman.mpdl.mpg.de/pubman/item/escidoc:2140623/component/escidoc:2140622/IPP_1_338.pdf

${ }^{16}$ I.G.J.Classen, et.al., Rev.Sci.Instr.81 (2010) 10D929

${ }^{17}$ S. K. Rathgeber, Plasma Phys. Control. Fusion 55 (2013) 025004

${ }^{18}$ V. Igochine et. al. Physics of Plasmas, 21, 110702 (2014)

${ }^{19}$ A. Gude, Chapter 3 in V. Igochine (ed.) "Active Control of Magneto-hydrodynamic Instabilities in Hot Plasmas" (Springer Series on Atomic, Optical, and Plasma Physics, Vol 83, 2015)

${ }^{20}$ J.P. Freidberg, "Ideal MHD", Cambrige University Press 2014, Chapter 11.6

${ }^{21}$ H. Zohm, "Magnetohydrodynamic Stability of Tokamaks", Wiley-VCH, 2015, p.56-p.59

${ }^{22} \mathrm{Q}$. Yu, to be published

${ }^{23}$ D.Vezinet et.al. 42nd EPS Conference on Plasma Physics, 2015 Lisbon, Portugal, P1.138

${ }^{24}$ D.Vezinet et.al., Nuclear Fusion, 086001, (2016)

${ }^{25}$ J. Wesson, Plasma Phys. Control. Fusion Vol.28, (1986) p.243

${ }^{26}$ H.A. Holties et.al., Nuclear Fusion, Vol. 36, No. 8 (1996) p.974

${ }^{27}$ I.T. Chapman et. al., Nucl. Fusion 50 (2010) 045007

${ }^{28}$ V. Igochine et.al. 42nd EPS Conference on Plasma Physics, 2015 Lisbon, Portugal, P1.144

${ }^{29}$ J. Meskat et.al., Plasma Phys. Control. Fusion 43 (2001) 1325-1332

${ }^{30}$ P. L. Pritchett, et.al. Physics of Fluids 23, 1368 (1980)

${ }^{31}$ P.J. Mc Carthy et al., Plasma Phys. Control. Fusion 54(2012) 015010

${ }^{32}$ E. Strumberger et al, Journal of Physics: Conference Series 561 (2014) 012016

${ }^{33}$ E.Strumberger, et.al., Nucl. Fusion 54 (2014) 064019

${ }^{34}$ W.A. Cooper, et.al., Phys. Rev. Lett. 105 (2010) 035003

${ }^{35}$ E.Prist, T. Forbes, „Magnetic reconnection“, Cambrige University Press, 2000, (Chapter 4) 